\title{
Panorama de la investigación y desarrollo de las nanotecnologías para el tratamiento de agua en México
}

\section{Outlook of research and development of nanotechnologies for water treatment in Mexico}

\author{
José Domingo Rafael Castañeda Olvera ${ }^{1}$ \\ Universidad Tecnológica Fidel Velázquez, México \\ Guillermo Foladori ${ }^{2}$ \\ Universidad Autónoma de Zacatecas, México
}

\author{
Sein León Silva ${ }^{3}$ \\ Instituto Politécnico Nacional, México \\ Eduardo Robles-Belmont ${ }^{4}$ \\ Instituto Politécnico Nacional, México
}

\author{
Edgar Záyago Lau $^{5}$ \\ Universidad Autónoma de Zacatecas, México
}

\begin{abstract}
Resumen. El objetivo de este artículo es revisar el panorama acerca de las nanotecnologías para el tratamiento del agua en México, desde la investigación y desarrollo hasta los procesos y dispositivos disponibles en el mercado. El estudio abarcó un análisis bibliométrico de publicaciones científicas, una base de datos con los grupos de investigación sobre el tema, un catálogo de patentes y, finalmente, una descripción de las empresas mexicanas que producen instrumentos y dispositivos para descontaminar, purificar y desalinizar el agua. Pese a que no existe una estrategia nacional respecto a las nanotecnologías en nuestro país, de 2000 a 2015, se publicaron 115 artículos sobre el tema en 45 instituciones, lo cual coloca a México como país que aporta gran porcentaje de la investigación sobre el tema en la región; asimismo, hay empresas nacionales e internacionales que ofrecen opciones para el tratamiento del agua, a saber: dispositivos y materiales nanoestructurados, así como servicios para purificar el agua con nanopartículas.
\end{abstract}

Palabras clave. Nanotecnología, tratamiento de agua, recurso acuífero, innovación tecnológica, México

\begin{abstract}
The aim of this article is to review the outlook about nanotechnologies for water treatment in Mexico, from research and development to the processes and devices available in the market. The study included a bibliometric analysis of scientific publications, a database with research groups on the subject, a catalog of patents, and, finally, a description of Mexican companies that produce instruments and devices to decontaminate, purify and desalinate water. Although there is no national strategy regarding nanotechnologies in our country, from 2000 to 2015,115 articles on the subject were published in 45 institutions, placing Mexico as a country that contributes a large percentage of research on the subject; also, there are national and international companies that offer different options for water treatment, from nanostructured devices and materials to services for water purification with different nanoparticles.
\end{abstract}

Keywords. Nanotechnology, water treatment, water resource, technological innovation, Mexico

\footnotetext{
${ }^{1}$ José Domingo Rafael Castañeda Olvera. Doctor en Sociología, Universidad Autónoma Metropolitana, México. Posdoctorado en Unidad de Estudios del Desarrollo, Universidad Autónoma de Zacatecas. División Académica de Tecnología Ambiental y Nanotecnología, Universidad Tecnológica Fidel Velázquez. Correo electrónico: rafaelcastaneda10@yahoo.com.mx

${ }^{2}$ Guillermo Foladori. Doctor en Economía por la Universidad Nacional Autónoma de México. Unidad de Estudios del Desarrollo, Universidad Autónoma de Zacatecas. Correo electrónico: gfoladori@gmail.com

${ }^{3}$ Sein León Silva. Doctorante en el Programa Transdisciplinario en Desarrollo Científico y Tecnológico para la Sociedad del Centro de Investigaciones y de Estudios Avanzados del Instituto Politécnico Nacional. Correo electrónico: sleon@cinvestav.mx

${ }^{4}$ Eduardo Robles-Belmont. Doctor en Sociología Industrial, Universidad de Grenoble (Francia). Investigador Asociado en el Departamento de Modelación Matemática de Sistemas Sociales del Instituto de Investigaciones en Matemáticas Aplicadas y en Sistemas de la UNAM. Correo electrónico: roblesbelmont@yahoo.fr

${ }^{5}$ Edgar Záyago Lau. Doctor en Estudio del Desarrollo, Universidad Autónoma de Zacatecas. Postdoctorado en la Universidad de California, Santa Bárbara. Docente investigador de la Unidad Académica de Estudios del Desarrollo de la Universidad Autónoma de Zacatecas. Correo electrónico: zayagolau@gmail.com
} 


\section{Introducción}

Los problemas relacionados con la contaminación de los cuerpos de agua tienen alcance global. Varios factores, como el crecimiento demográfico, la urbanización y la industrialización (asociados con un aumento de la producción y del consumo), han colocado en estrés continuo a los recursos hídricos del planeta (UNESCO, 2016). El creciente uso de fertilizantes y de materiales químicos es otro factor que ha contribuido a la eutrofización de los ríos y la generación de zonas muertas en diferentes hábitats (UN-Water, 2016). La mala gestión de las aguas residuales, así como la falta de políticas públicas también han complicado este escenario (Corcoran et al., 2016). Los procesos socioeconómicos de empobrecimiento y la tendencia a la privatización de las fuentes de agua y de los sistemas de distribución son factores profundos que también abonan a la actual crisis mundial del agua.

Se trata de un problema complejo, multidimensional y de difícil resolución, que requiere atender las causas socioeconómicas y los aspectos legales, administrativos y de planeación, además de las alternativas tecnológicas para revertir situaciones existentes de contaminación y de acceso al agua. Este artículo ilustra sobre este último aspecto. Uno de los avances más destacados de los años recientes, en materia de ciencia y tecnología, son las nanotecnologías. Estas, también llamadas tecnologías convergentes, se hallan prácticamente en todas las áreas de investigación, y sus productos se encuentran ya en el mercado.

La presente pesquisa aporta información al debate sobre investigación y desarrollo del tema, así como un análisis de empresas que comercializan instrumentos y dispositivos nanotecnológicos para el tratamiento del agua en México.
México es la undécima economía más grande del mundo -según el producto interno bruto ajustado a la paridad de poder adquisitivo (FMI, 2017), una potencia industrial emergente, $y$, naturalmente, en constante demanda de agua-. A pesar de ser un tema prioritario, la escasez de agua y la contaminación es un asunto pendiente en la agenda del gobierno mexicano.

De acuerdo con la Comisión Nacional del Agua (CONAGUA), siete de las 13 regiones hidrológico-administrativas en las que se divide el territorio nacional sufren de un alto grado de estrés hídrico (CONAGUA, 2015). 77\% de la población nacional se concentra en regiones donde solo se dispone de $31 \%$ de fuentes de agua viables para consumo (CONAGUA, 2015). El Consejo Consultivo del Agua (CCA) afirma que $2 / 3$ de nuestro territorio se considera árido o semiárido, y casi $80 \%$ de la población del país se concentra en esa zona (CCA, 2017). Según estimaciones de CONAGUA existe una sobreexplotación de las reservas subterráneas que, actualmente, abastecen más de $28 \%$ de la demanda. La región que corresponde al Valle de México, donde se concentra una gran parte de la actividad industrial del país y donde se presenta el mayor índice poblacional por metro cuadrado, experimenta un estrés crítico constante, ya que existe un desequilibrio entre la disponibilidad media del agua y la demanda de este recurso; y se espera un aumento de 2.6 millones de habitantes para el año 2030, cuyo acceso al recurso hídrico estará sujeto a una limitada disponibilidad (CONAGUA, 2015).

La respuesta gubernamental ante tal escenario ha sido insuficiente, debido a que las estrategias y políticas públicas encaminadas a solucionar esta problemática no han sido eficaces. De ahí que se busquen respuestas en la esfera científica misma. Uno de los paquetes tecnológicos más recientes, destinado a tratar los cuerpos de agua contaminados, 
a potabilizar agua y a mejorar los procesos de desalinización, son las nanotecnologías.

Las nanotecnologías son el conjunto de ciencias que se encargan del estudio, diseño y manipulación de la materia a escala nanométrica $\left(10^{-9}\right)$. A esta escala los materiales manifiestan propiedades físico-químicas novedosas respecto de los mismos materiales en escala mayor; así, los nanomateriales presentan propiedades de reactividad, magnetismo, conductividad, resistencia, etcétera, mejorados, lo que hace más eficiente su uso y aplicación en diversas áreas industriales. A partir del reordenamiento de átomos y de moléculas, las nanotecnologías nos permitirán la posibilidad de fabricar nuevos materiales, dispositivos, aparatos y sistemas de menor costo y con propiedades únicas.

Las nanotecnologías forman parte de una nueva plataforma tecnológica con capacidad para mejorar los métodos de tratamiento de cuerpos de agua contaminados. Varios países están aplicando procesos basados en nanotecnología para resolver problemas relacionados con el vital líquido. Diferentes aplicaciones nanotecnológicas hacen uso de estas capacidades novedosas para el tratamiento del agua (Kharisov et al., 2012).

Este artículo sistematiza los resultados de una investigación tendiente a mostrar el estado del arte de las nanotecnologías aplicadas al agua en México. Infelizmente, no existe un registro oficial sobre esta temática, de manera que la pesquisa recopiló, ordenó y sistematizó información de diversasfuentes.Losresultadossondeinteréspara funcionarios gubernamentales, investigadores, estudiantes y para organizaciones de la sociedad civil y empresas, ya que pueden alertar sobre alternativas no suficientemente divulgadas.

Desarrollo de las nanotecnologías en México

Según investigaciones previas que han considerado la evolución de las patentes, de empresas, de recursos humanos einfraestructura, y deartículoscientíficospublicados, Méxicoocupa el segundo lugar en Latinoamérica, después de Brasil, en el desarrollo de las nanotecnologías (Invernizzi et al., 2015; Foladori et al., 2015; Záyago et al., 2014). Hay varios artículos que analizan el desarrollo de las nanotecnologías en México, pero prácticamente nada respecto de las aplicaciones al agua (Saldivar \& Walsh, 2015).

La Secretaría de Economía, en colaboración con el Centro de Investigación de Materiales Avanzados (CIMAV), realizó un estudio sobre las nanotecnologías en México, donde ilustró que, entre 1998 y 2004, el gobierno mexicano, a través del Consejo Nacional de Ciencia y Tecnología (CONACyT), apoyó un total de 152 proyectos de investigación por valor de $\$ 14.4$ millones (CIMAV/SE, 2008). Takeuchi y Mora (2011) estimaron que el total de fondos para investigación y desarrollo en el ramo, entre 2006 y 2009, ascendió a unos 60 millones de dólares.

Por otra parte, como subapartado del Plan Nacional de Desarrollo 2007-2012, se instauró el Gasto Nacional en Ciencia, Tecnología e Innovación (GNCTI), con el objetivo de apoyar el desarrollo de nuevas plataformas tecnológicas (Conacyt, 2014); sin embargo, no hay datos desagregados para nanotecnología.

La política pública de desarrollo de las nanotecnologías en México se ha concentrado en tres áreas: la creación de redes de investigación, los laboratorios nacionales y los clústeres industriales (Záyago et al., 2014).

\section{Metodología}

La literatura internacional especializada en aplicaciones de nanotecnología al agua suele establecer tres categorías que corresponden con los diferentes usos: remediación y purificación; detección de contaminantes y filtración; y desalinización (Rickerby y Morrison, 2016; Veseashta et al., 2016; Qu et al., 2013; 
Prachi et al., 2016; Bora y Dutta, 2016). Estas aplicaciones se implementan, a veces, de manera simultánea o en combinación, lo cual dificulta la individualización en algunos casos, pero la clasificación fue creada para detectar el uso potencial del agua ${ }^{\mathrm{i}}$.

Para obtener información, se implementaron varias estrategias de búsqueda. La primera consistió en la identificación de publicaciones científicas, mediante un análisis bibliométrico y a partir de la base de datos de la Web of Science (WoS) para el periodo 2000-2015. Se identificaron aquellos artículos donde al menos un autor estuviese vinculado a una institución mexicana. Las palabras clave aplicadas al título, resumen y palabras clave del artículo pueden verse en el Anexo 1.

La segunda estrategia de búsqueda fue para identificar grupos de investigación sobre el tema. Para ello, se analizaron los Cuerpos Académicos (CA) del Programa de Desarrollo Profesional para Profesores (PRODEP) de la Secretaría de Educación Pública (SEP). Los CA del PRODEP constituyen un programa que apoya la formación de grupos de investigación en torno a temas específicos. Básicamente, este es un catálogo de investigadores afiliados a instituciones públicas de educación superior en México.

Existen varias instituciones afiliadas al programa PRODEP, como universidades públicas del Estado, universidades politécnicas, universidades tecnológicas, institutos tecnológicos federales, institutos tecnológicos

${ }^{\mathrm{i}}$ En el tratamiento y la remediación, las nanotecnologías tienen el potencial para mejorar la eficiencia del tratamiento de agua: floculación, sedimentación, coagulación y carbón activado (Li et al., 2015; Hosseinpour et al., 2015; Qu et al., 2013; Brame et al., 2016; Ahmed et al., 2016). Algunas nanopartículas son muy eficaces en la captura de metales pesados, lo cual facilita su eliminación de las masas de agua. Para la filtración, las nanotecnologías tienen el potencial de desarrollar dispositivos capaces de detectar y de controlar la contaminación del agua, acelerando el tránsito para su remediación o purificación (Qu et al., 2016; El-Safty et al., 2016; Pendergast et al., 2016; Pronovost, 2016). Para la desalinización, los nanoprocesos prometen disminuir los costos, por ejemplo, mediante el uso de membranas con nanopartículas de plata y de zinc (Baruah et al., 2016; Seul-Yi y Park, 2016). descentralizados, politécnicos y escuelas normales. Esta estrategia de búsqueda se ha utilizado en otros procesos de investigación y para otros temas, ya que representa un proceso simplificado en la recopilación de información (Záyago et al., 2015). El Anexo 2 ilustra los filtros (palabras clave) empleados para procesar los datos de la página web de PRODEP (Gobierno de la República, s.f.).

La tercera estrategia de búsqueda fue para identificar las principales instituciones de educación superior que no forman parte del PRODEP. La lista comprende: la Universidad Nacional Autónoma de México (UNAM); el Instituto Politécnico Nacional (IPN); la Universidad Autónoma Metropolitana (UAM); todos los centros de investigación del CONACyT; y las principales instituciones privadas. Los datos obtenidos se registraron en una base de datos que incluye los siguientes campos: nombre de la institución, nombres de los investigadores, área de investigación de interés (nano y agua) y dirección de correo electrónico.

La cuarta estrategia de búsqueda fue para identificar las solicitudes de patente registradas en la Organización Mundial de la Propiedad Intelectual (OMPI) para México. Después de explorar la base de datos Patentscope de la OMPI, la información obtenida fue clasificada en las mismas categorías (remediación y purificación, detección de contaminantes y filtración, y desalinización). Cabe señalar que el procedimiento de registro de solicitudes y patentes difiere de un país a otro y de un representante legal a otro. Los resultados pueden variar entre las bases de datos, pero la OMPI concentra la mayoría de los registros de patentes. Después, fue realizado un filtrado manual de cada registro para eliminar duplicados o registros que no estaban relacionados con el tema. El Anexo 3 resume los términos de búsqueda de las patentes. 
La quinta estrategia de búsqueda fue para identificar las empresas mexicanas que fabrican productos nanotecnológicos orientados a la remediación/purificación, detección/filtración o desalinización del agua. Esto se desarrolló a partir de un inventario existente de empresas de nanotecnología en México (Záyago et al., 2014). La base de datos completa está disponible en la página web nanoeconomía en México (CINVESTAV, 2016a). Es necesario advertir que el número de empresas varía continuamente; por lo tanto, los datos señalados en este artículo corresponden al registro de 2014.

\section{Resultados}

\section{Las nanotecnologías representan una} plataforma tecnológica cuya capacidad permite que ciertos métodos de tratamiento de agua sean más eficientes. El hecho de que la investigación aquí resumida haya cubierto desde la investigación y desarrollo, mediante artículos científicos publicados, grupos de investigación y patentes, hasta empresas productoras de instrumentos y de dispositivos da una visión general del estado de las nanotecnologías en relación con el agua en México, incluyendo la inserción productiva y su potencial impacto.

El análisis bibliométrico registró 117 artículos científicos con al menos un autor afiliado a una institución mexicana en el total del periodo 2000-2015. Se clasificaron los artículos según la categoría. Los resultados se muestran en las figuras 1 y 2.

En este orden de ideas, solo 3\% de los artículos están orientados a desalinización. La filtración

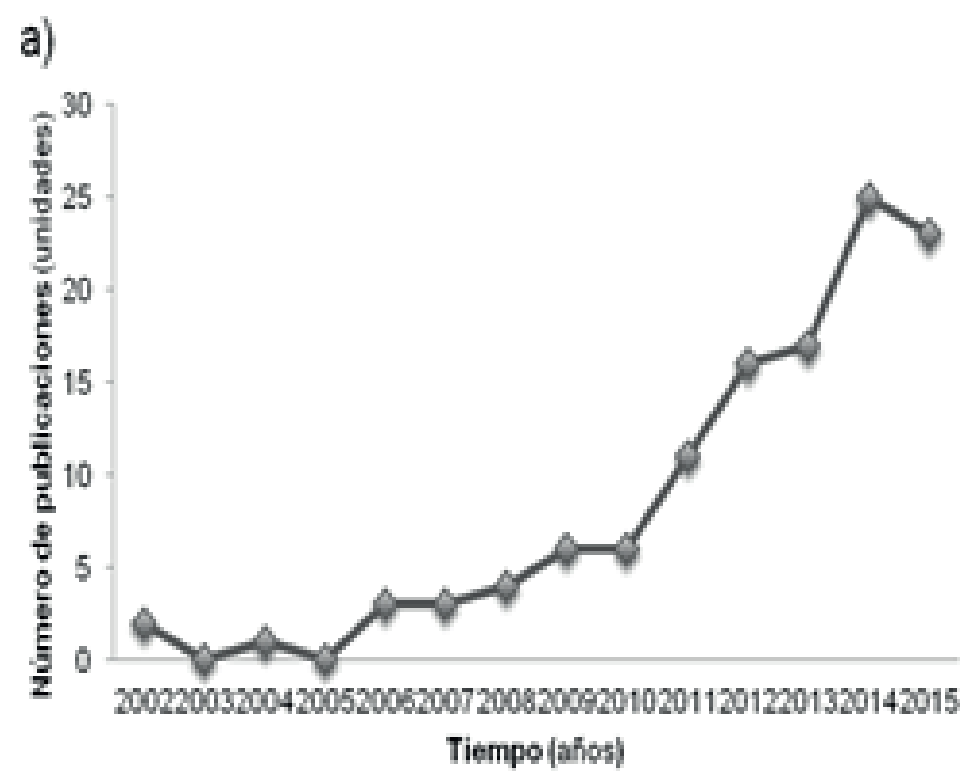

Figura 1. Número de artículos de acuerdo al área de aplicación de nanotecnologías y agua, período 2002-2015. Nota. Elaborado por Rafael Castañeda, Guillermo Foladori, Eduardo Robles-Belmont, Sein León Silva y Edgar Záyago a partir de la base de datos de la Web of Science (WoS) para el periodo 2000-2015. 


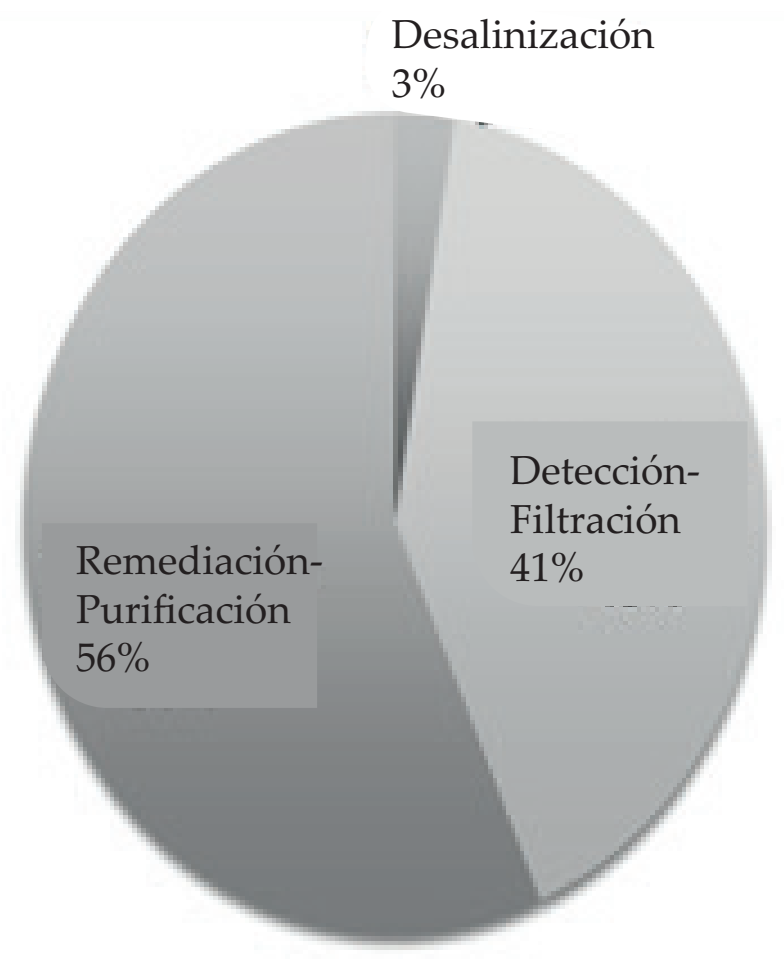

Figura 2. Porcentaje de artículos de acuerdo al área de aplicación de nanotecnologías y agua.

Nota. Elaborado por Rafael Castañeda, Guillermo Foladori, Eduardo Robles-Belmont, Sein León Silva y Edgar Záyago a partir de la base de datos de la Web of Science (WoS) para el periodo 2000-2015.

y la detección representa $41 \%$ del total. El área de aplicación con los más publicados fue el de remediación y purificación con 56\%. En esta última área, la mayoría de la investigación y experimentación hizo uso de nanotubos de carbono, así como de plata, oro, óxido de hierro y nanomateriales de magnetita para eliminar contaminantes.

Adicionalmente, se analizaron las redes de colaboración de las diferentes instituciones relacionadas con los artículos científicos, incluyendo instituciones internacionales. Los resultados se aprecian en la figura $3^{\text {ii }}$.

ii Se utilizaron los software Pajek y VOSviewer, que facilitan la visualización y el análisis de datos.
El tamaño de cada nodo determina la frecuencia de participación de cada institución. Los nodos verdes representan las instituciones extranjeras; y los rojos, la contraparte mexicana. Cada borde detalla la relación de coautor entre las instituciones. La UNAM tiene el mayor número de publicaciones (37), seguido por el CIMAV (25), el Instituto Nacional de Investigación Nuclear (ININ) (11) y el Instituto Potosino de Investigación Científica y Tecnológica (IPICyT) (10).

Estos números están directamente relacionados con el apoyo financiero que el gobierno mexicano asigna a algunas instituciones educativas centrales de nuestro 
Panorama de la investigación y desarrollo de las nanotecnologías para tratamiento de agua

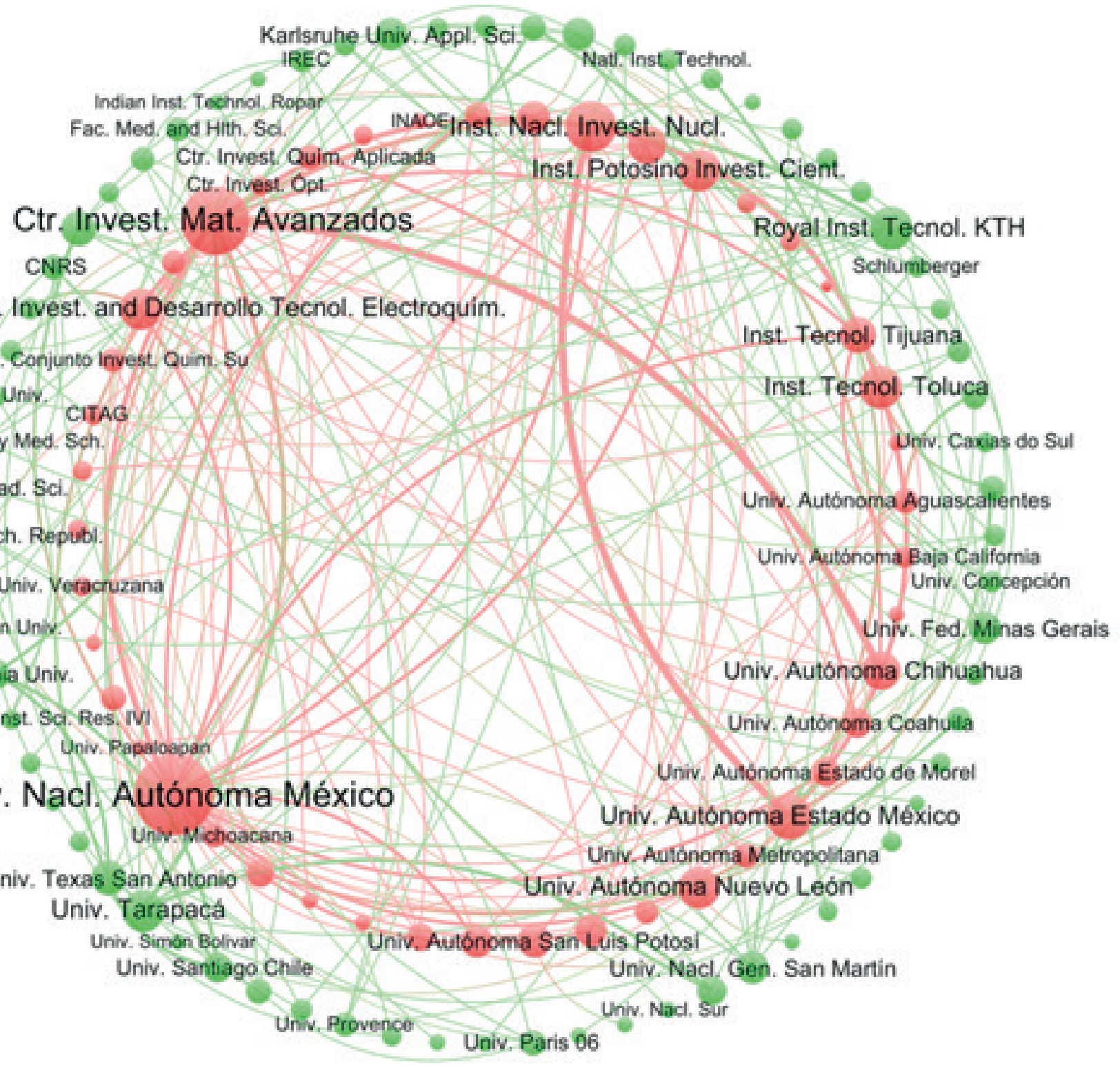

Figura 3. Redes de investigación en nanotecnología y agua en México.

Nota. Elaborado por Rafael Castañeda, Guillermo Foladori, Eduardo Robles-Belmont, Sein León Silva y Edgar Záyago con información de la base de datos "Nanociencias y Nanotecnologías" del Laboratorio de Redes, IIMAS, UNAM. 
país, así como a centros de investigación especializados. Estas instituciones también cuentan con una infraestructura de difusión más eficaz que las de los institutos medianos/ regionales o las universidades estatales.

En total, se encontraron 45 instituciones mexicanas con publicaciones en nanotecnologías para agua; sin embargo, la mayoría publicó menos de tres artículos en el período analizado. Las cinco instituciones con el mayor número, según las categorías, pueden verse en la tabla 1.

En cuanto a los países con mayor colaboración, los Estados Unidos encabezan la lista con nueve artículos; España figura en el segundo lugar con ocho; Francia en tercer lugar con siete y Chile con seis (figura 4).

El análisis de los grupos de investigación incluye los 14 grupos registrados por los CA de PRODEP y otros 27 distribuidos en instituciones de investigación que no forman parte del PRODEP. La distribución de los de PRODEP, según las categorías investigadas, puede verse en la tabla 2.
Puede notarse que existe una distribución proporcional en la cantidad de grupos de investigación destinados a las dos primeras categorías; no obstante, falta el grupo de investigación de PRODEP dedicado a la categoría de desalinización.

Los otros 27 grupos que incluyen muchas de las principales universidades y varios centros de investigación de primer nivel, pero que no integran PRODEP, están distribuidos por las categorías e instituciones, según la figura 5.

Varias de estas instituciones disponen más de un grupo de investigación. El IPN cuenta con 13; la UNAM, con seis; el IPICyT, con 4; la Universidad Autónoma de Yucatán, con dos; el Instituto Tecnológico de Estudios Superiores de Monterrey, con uno; y la Universidad de las Américas, también con un grupo.

Si sumamos los grupos de investigación de PRODEP y estas otras instituciones, la mayoría de ellos, 29 grupos de un total de 41, están relacionados con el análisis de materiales nanoestructurados, su caracterización y posibles

Tabla 1

Principales instituciones mexicanas con publicaciones de nanotecnología y agua por categoría

\begin{tabular}{lccc}
\hline$\quad$ Institución & $\begin{array}{c}\text { Remediación } \\
\text { Purificación }\end{array}$ & $\begin{array}{c}\text { Detección } \\
\text { Filtración }\end{array}$ & Desalinización \\
\hline UNAM & 23 & 14 & \\
ININ & 9 & & \\
CIMAV & 9 & 6 & \\
UAEM & 7 & & \\
IPN & 7 & 9 & \\
IPICYT & & 4 & 1 \\
Inst. Tecnológico de Toluca & & 4 & 1 \\
UASLP & & & \\
Inst. Tecnológico de Sonora & & & \\
UAQ & & & \\
\hline
\end{tabular}

Fuente. Datos de Web of Science Collection hasta julio de 2016. 
Panorama de la investigación y desarrollo de las nanotecnologías para tratamiento de agua

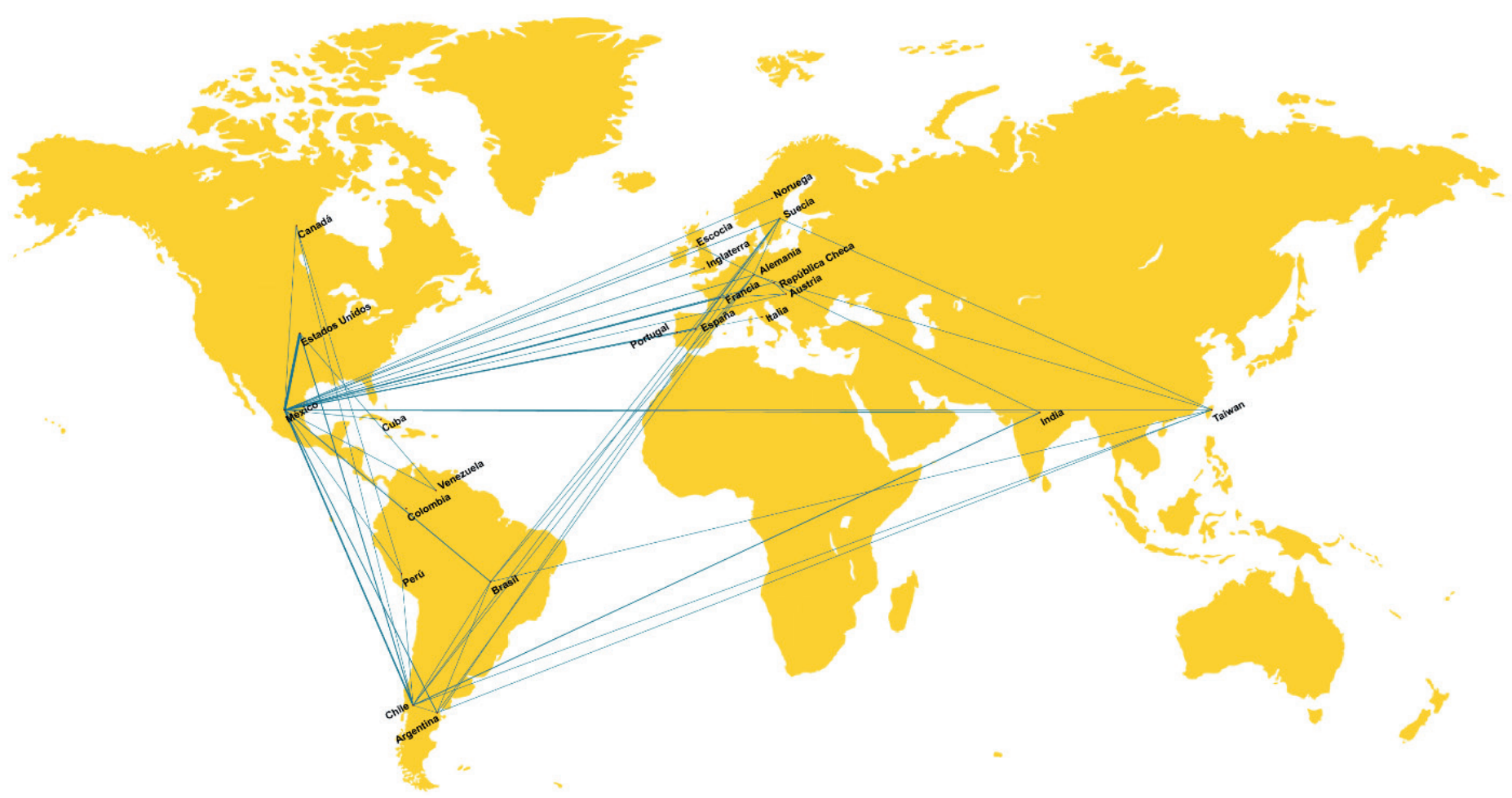

Figura 4. Redes de colaboración con otros países en nanotecnologías y agua.

Nota. Elaborado por Rafael Castañeda, Guillermo Foladori, Eduardo Robles-Belmont, Sein León Silva y Edgar Záyago con información de la base de datos "Nanociencias y Nanotecnologías" del Laboratorio de Redes, IIMAS, UNAM.

Tabla 2

Los Cuerpos Académicos de PRODEP de investigación y desarrollo en nanotecnología y agua

\begin{tabular}{|c|c|c|c|c|}
\hline Institución & Departamento & $\begin{array}{l}\text { Remediación } \\
\text { Purificación }\end{array}$ & $\begin{array}{l}\text { Detección } \\
\text { Filtración }\end{array}$ & Desalinización \\
\hline $\begin{array}{l}\text { Instituto Tecnológico de Ciudad } \\
\text { Madero }\end{array}$ & $\begin{array}{c}\text { Nanotecnologías y Energías } \\
\text { Renovables }\end{array}$ & $X$ & & \\
\hline Instituto Tecnológico de Oaxaca & $\begin{array}{l}\text { Ciencia de los Materiales y } \\
\text { Química Ambiental }\end{array}$ & & $X$ & \\
\hline Instituto Tecnológico de Toluca & $\begin{array}{c}\text { Desarrollo Nanotecnológico y } \\
\text { Evaluación de Materiales para } \\
\text { Aplicaciones Ambientales }\end{array}$ & $X$ & $X$ & \\
\hline
\end{tabular}

Continúa... 
Universidad Autónoma de Sinaloa

Universidad Autónoma

Metropolitana Azcapotzalco

Universidad de Guadalajara

Centro Universitario Ciénega

Universidad Tecnológica Fidel

Velázquez

Universidad Tecnológica de

Tula-Tepeji

Instituto Tecnológico de Celaya

Instituto Tecnológico de Valle

de Morelia

Instituto Tecnológico Superior

de Irapuato

Universidad Autónoma de

Morelos

Universidad Autónoma

Metropolitana Azcapotzalco

Universidad Autónoma

Metropolitana Lerma
Cristales e Ingeniería

Ambiental

Nanotecnología y Calidad Ambiental

Nanomateriales Polímeros y catalizadores

Nanotecnología

Ingeniería y Sistemas

Ambientales

Química de Nanomateriales

Bioingeniería en agronomía sustentable

Micro- y Nanociencias

Diseño y Caracterización de

Nuevos Materiales Aplicables

en Ingeniería Ambiental

Ingeniería y Aplicaciones de

Materiales Nanoestructurados

Materiales Nanoestructurados
$X$

X $\quad X$

$X$

$X$

X $\quad X$

$X$

$X$

$X$

$X$

X $\quad X$

$X \quad X$

Fuente. Elaborado por Rafael Castañeda, Guillermo Foladori, Eduardo Robles-Belmont, Sein León Silva y Edgar Záyago a partir de los datos de los CA del PRODEP (2016).
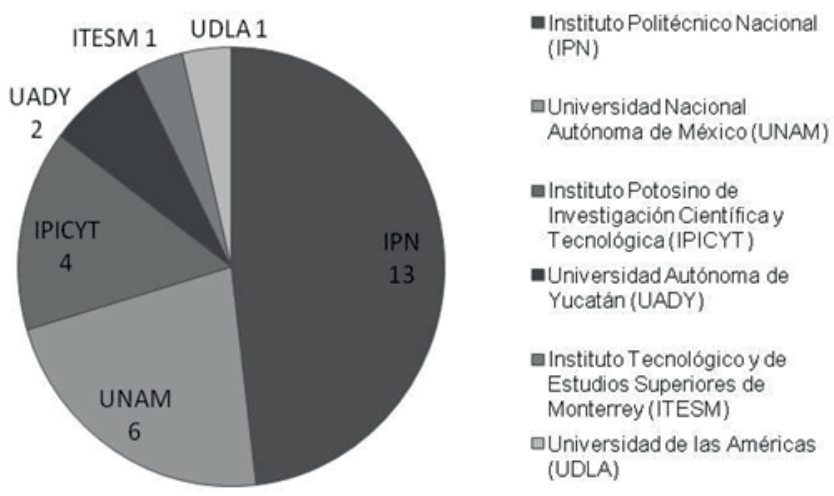

Figura 5. Grupos de investigación en nanotecnología y agua que no integran el programa de PRODEP.

Nota. Elaborado por Rafael Castañeda, Guillermo Foladori, Eduardo Robles-Belmont, Sein León Silva y Edgar Záyago a partir de los datos de los CA del PRODEP obtenidos. 
aplicaciones para remediación y purificación de cuerpos de agua. Veinte de ellos se centran en el análisis de materiales nanoestructurados para acelerar los métodos de limpieza.

La mayoría del resto de los grupos investiga en detección y filtración, y solo existen dos grupos orientados a desalinización.

En cuanto a la sección de patentes, identificamos un total de 60 solicitudes sobre aplicaciones de nanotecnología y agua en México: 33 pertenecen al campo de filtración y detección; 22 se relaccionan con sistemas o procesos para remediación y purificación; y cinco, con desalinización. En cierta forma, reproducen la distribución de las categorías por grupo de investigación (figura 6).

Del total de 60 patentes con cobertura para México, solo cuatro corresponden a inventores mexicanos; una, al Centro de Investigación y Desarrollo de Alimentos (CIAD); otra, al Instituto Tecnológico Nacional de México de la SEP; y las dos restantes, a inventores sin vínculos con instituciones.

En cuanto a las empresas e instituciones extranjeras, Degremont, Toray Industries, Indiana Institute of Technology, Pure Water Purification y Siemens Water Technologies (adquirida por Evoqua water), tienen entre cuatro y dos patentes, el resto una (figura 7). Además, se aprecia una distribución equitativa entre las patentes destinadas a filtración y a detección de contaminantes (figura 7a) y las destinadas a purificación y a remediación (figura $7 \mathrm{~b}$ ). Apenas dos patentes están dedicadas a desalinización (figura 7c).

El inventario de empresas de nanotecnología en México solo identifica cinco que trabajan con agua (Záyago et al., 2014). Dos están situadas en la ciudad de México; dos, en Monterrey; y una, en

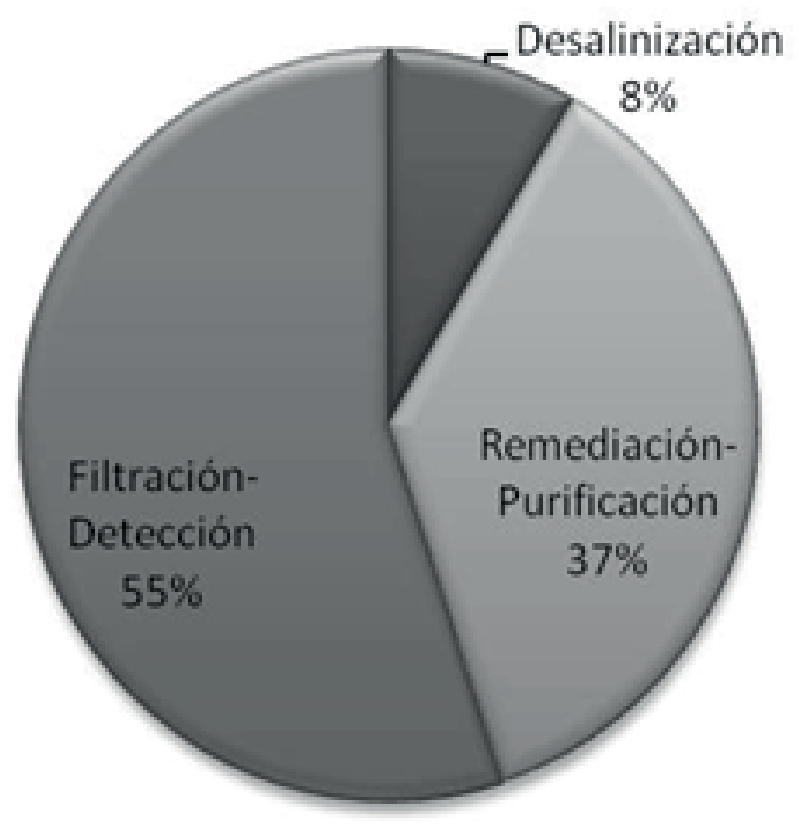

Figura 6. Patentes de nanotecnologías y agua en México.

Nota. Elaborado por Rafael Castañeda, Guillermo Foladori, Eduardo Robles-Belmont, Sein León Silva y Edgar Záyago de acuerdo a los datos obtenidos de las solicitudes de patente registradas en la Organización Mundial de la Propiedad Intelectual (OMPI) para México y de la base de datos Patentscope de la OMPI obtenidos. 
a) Detección y filtración

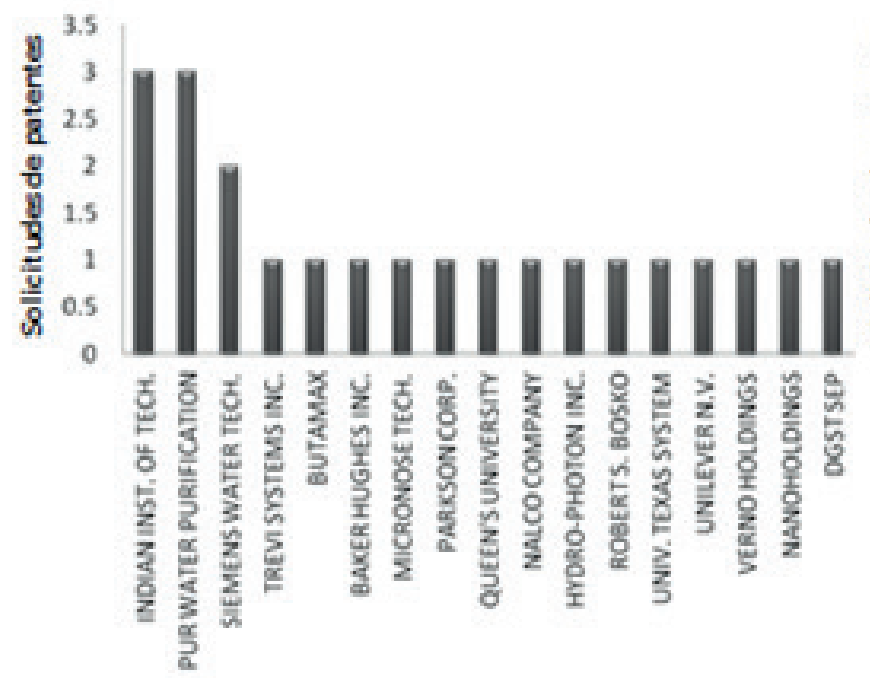

c) Desalinización

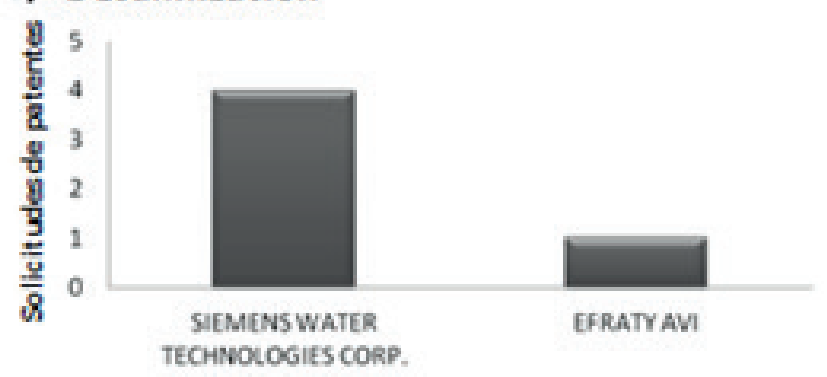

b) Purificación y remediación

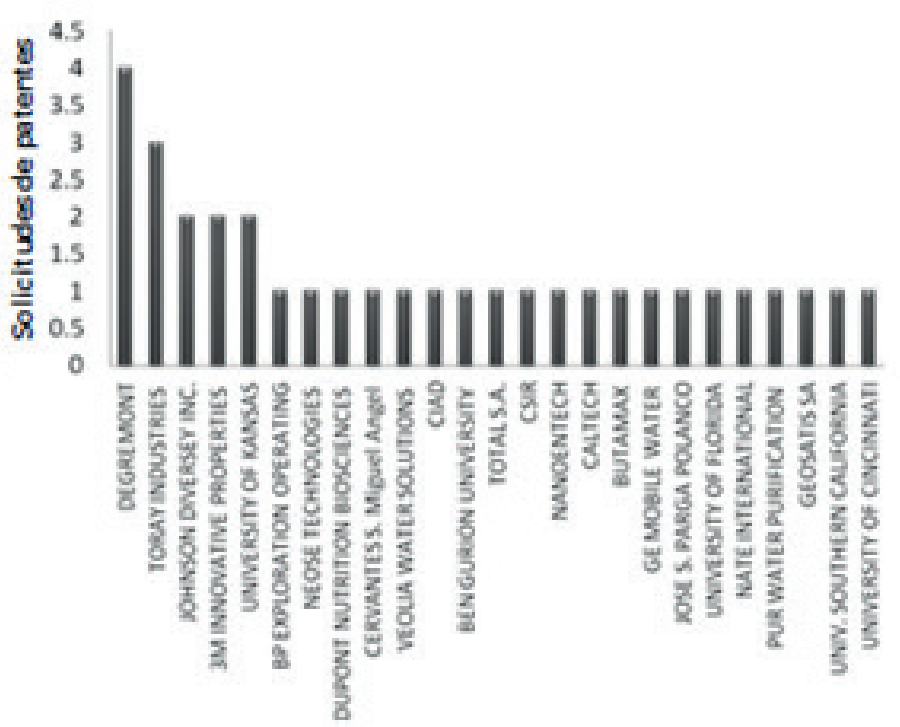

Figura 7. Empresas e instituciones extranjeras con patentes en nanotecnología y agua en México

Nota. Elaborado por Edgar Záyago, Sein León y Guillermo Foladori con base en las solicitudes de patente registradas en la Organización Mundial de la Propiedad Intelectual (OMPI) para México y de la base de datos Patentscope de la OMPI.

Guadalajara. Dichas empresas venden productos que aplican nanofiltración y sus instalaciones de manufactura se encuentran en México.

Nvironmental ACS, ubicada en la ciudad de México, diseña y fabrica sistemas de nanofiltración a medida, sistemas que simulan a las plataformas de ósmosis inversa (ACS, 2016). La nanofiltración es un método eficaz, por ejemplo, para tratar el agua contaminada con pesticidas y sustancias químicas altamente tóxicas basadas en colorantes. El equipo utilizado tiene diferentes configuraciones: membrana espiral, membrana tubular y membranas de fibra hueca. Estos sistemas capturan residuos orgánicos e inorgánicos, eliminándolos de los cuerpos de agua.

Carbotecnia se localiza en Guadalajara. Es una empresa mexicana especializada en el desarrollo y comercialización de carbón activado para el tratamiento de agua de uso doméstico, industrial y comercial. Ofrece equipos de alta tecnología con diferentes técnicas, incluye la nanofiltración (Carbotecnia, 2016).

Lobal Proventus se encuentra en Monterrey. Esta compañía fabrica equipos para el tratamiento de agua, válvulas industriales y equipos de bombeo. En cuanto al tratamiento 
Panorama de la investigación y desarrollo de las nanotecnologías para tratamiento de agua

del agua, ofrece sistemas de ósmosis inversa, nanofiltración y ultrafiltración, entre otros productos (Global Proventus, 2016). La empresa, según su sitio web, también exporta a Europa, América y Asia.

En la ciudad de México, también está situada la empresa Heltec, que fabrica equipos de tratamiento de agua. Su cartera incluye productos de ultrafiltración, nanofiltración, microfiltración y electrodesionización (Heltec, 2016). No está claro si esta proviene de capital mexicano o extranjero, ya que en su sitio web dice que comenzó a operar como representante de GE Water Technologies. Heltec es una corporación que ofrece sus productos en los Estados Unidos, Canadá, Irlanda, Inglaterra y Alemania, así como en América Central y América del Sur.

La quinta empresa es Aquapro, cuya sede está en Monterrey. Ofrece la instalación de equipos y de sistemas de tratamiento de agua. En cuanto a las aplicaciones nanotecnológicas, Aquapro fabrica equipos de nanofiltración. La empresa utiliza membranas de poliamida en una configuración en espiral y aplica nanopartículas para eliminar los contaminantes orgánicos e inorgánicos (fouling) (Aquapro, 2016).

\section{Conclusiones}

Larevisióndelpanoramasobrelainvestigación y el desarrollo de las nanotecnologías aplicadas al tratamiento del agua en México corrobora el importante papel que nuestro país tiene en la región respecto al tema. Nuestro país, al lado de Brasil y de Argentina, encabezan el impulso de las nanotecnologías en Latinoamérica en general. El número de artículos publicados, así como las redes de investigación consolidadas, configuran un escenario prometedor al respecto; sin embargo, este impulso sigue siendo insuficiente comparado con la magnitud de la problemática que la contaminación del agua en toda la región representa.
Si bien es cierto que la raíz de la solución al conflicto acuífero radica en la prevención de la contaminación y en la concientización sobre el cuidado del vital líquido, la opción que la esfera científica ofrece a los métodos de tratamiento de agua contaminada ha encontrado en las aplicaciones nanotecnológicas una ruta importante por explorar, ya que algunas nanopartículas aceleran tanto los procesos físico-químicos como los biológicos que se han utilizado tradicionalmente para el tratamiento de aguas contaminadas.

Siguiendo lineamientos internacionales, las aplicaciones de nanotecnologías al agua pueden dividirse a partir del uso final en tres categorías: purificación y remediación, filtración y detección de contaminantes, y desalinización. En las tres áreas, existe alguna presencia de investigaciones en México, aunque la mayoría de estas, así como sus posibles aplicaciones, se concentran en las primeras dos categorías anotadas. También, se destaca que un alto porcentaje de estas se concentran en los grandes centros de investigación, cuyas sedes se hospedan en urbes donde la contaminación acuífera tiene cierto tipo de características, hecho que se observa en la ruta de sus investigaciones: remediación y detección de contaminantes.

Por un lado, ese hecho resalta la ausencia de una estrategia gubernamental de alcance nacional alrededor de las nanotecnologías. Tal ausencia sesga la ruta de las investigaciones y desvincula las necesidades sociales con lo que las trayectorias científicas marcan. Por otro lado, hay una tendencia a criticar el uso de nanotecnologías para la remediación de agua y su purificación, ya que los escasos estudios acerca de toxicología de nanopartículas se incrementan, lo cual aumenta, de manera considerable, el riesgo para la salud humana y para el medio ambiente.

Ahora bien, los estudios nanotoxicológicos, se encuentran en ciernes, por eso, una ruta de 
investigación debería contemplar los impactos que el uso de nanotecnologías, en el tratamiento de cuerpos de agua, podrían traer consigo para el ser humano y el entorno.

En suma, la investigación aquí expuesta recoge datos sobre publicaciones científicas, grupos de investigación porinstitución, patentes yempresas con productos en el mercado. Hasta donde se sabe, es la primera investigación que recoge este tipo de información en México. Es posible que exista un desencuentro entre investigación, desarrollo y aplicaciones, ya que las patentes, que son uno de los indicadores intermedios, muestran que la mayoría de ellas corresponden a instituciones y a empresas extranjeras. Además, es muy reducido el número de empresas que producen instrumentos o dispositivos nanotecnológicos para el tratamiento del agua.

Sectores económicos de gran impacto social, como es el agua en México, deberían de tener instituciones públicas que registren los avances tecnológicos y sus potenciales aplicaciones. A falta de esta información, la investigación que aquí se presenta llena una laguna y sirve de base para futuras profundizaciones.

\section{Referencias}

ACS Medio Ambiente (2016). Nanofiltración. Recuperado de http://www.acsmedioambiente.com/nanofiltracion.html

Ahmed, T.; S. Imdad, K.; Yaldram, N.; Mohammad y P. Arshad (2016). Emerging Nanotechnology-Based Methods for Water $\mathrm{Pu}$ rification: A Review. Desalination and Water Treatment, 52(22-24), 4089-4101.

Aquapro (2016) Reverse Osmosis/nanofiltración. Recuperado de http://www.aquaproing. com/reverse.html

Baruah, S., K. Pal y J. Dutta (2016). Nanostructures Zinc Oxide for Water Treatment. $\mathrm{Na}$ noscience \& Nanotechnology-Asia, 2(2), 90-102.
Bora, T. y J. Dutta (2016). Applications of Nanotechnology in Wastewater Treatment. A Review. Journal of Nanoscience and Nanotechnology 14(1), 613-626.

Brame, J.; Q. Li y P. Álvarez (2011). Nanotechnology-enabled water treatment and reuse: emerging opportunities and challenges for developing countries. Trends in Food Science \& Technology, 22(11), 618-624.

Carbotecnia (2016). What is nanofiltration? Recuperado de http://www.carbotecnia.info/ encyclopedia/nanofiltration/

Centro de Investigación en Materiales Avanzados (CIMAV) y Secretaría de Energía (SE) (2008). Diagnóstico y Prospectiva de la Nanotecnología en México. México: CIMAV, SE.

Centro de Investigaciones y Estudios Avanzados (CINVESTAV) (2016). Nanoeconomía en México. Recuperado de http://micrositios. cinvestav.mx/nano/

CONACyT (Consejo Nacional de Ciencia y Tecnología) (2014). Informe General del Estado de la Ciencia, la Tecnología y la Innovación. México: Conacyt.

CCA (Consejo Consultivo del Agua). Panorama del agua en México. Recuperado de http:// www.aguas.org. $\mathrm{mx} / \mathrm{sitio} / \mathrm{index}$.php/panorama-del-agua/agua-en-mexico.

CONAGUA (Comisión Nacional del Agua) (2015). Num3ragua. México: Semarnat.

Corcoran, E.C.; E. Nellemann; R. Baker; D. Bos y H. Osborn (eds.) (2016). Sick Water? The central role of wastewater management in sustainable development. A Rapid Response Assessment. Nueva York: United Nations Environment Program, UN-HABITAT, GRID-Arendal. Recuperado de http://www. unep.org/pdf/ SickWaterscreen.pdf

El-Safty, S.; A. Shahat; Md. Rabiul y M. Mekawy (2011). Large Three-Dimensional 
Mesocage Pores Tailoring Silica Nanotubes as Membrane Filters: Nanofiltration and Permeation Flux of Proteins. Journal of Materials Chemistry, 21, 5593-5603.

Foladori, G.; E. Arteaga; E. Záyago; R. Appelbaum; E. Robles-Belmon; L. Villa, R. Parker y V. Leos (2015). Nanotechnology in Mexico: Key Findings Based on OECD Criteria. Minerva, 53(3), 279-301. DOI: 10.1007/s11024015-9281-6.

FMI (Fondo Monetario Internacional) (2017). Reporte para países seleccionados (ASPX). World Economic Outlook Database. Recuperado de www.imf.org.

Global Preventus (2016). Membranes. Recuperado de http://www.global-proventus.com/ productos/membranas/membranas/

Gobierno de la República (2018). https://promep.sep.gob.mx/ca1/

Heltec (2016). Membrane Technology. Recuperado de http://www.heltec.com.mx/ membranas.html.

Hosseinpour, M.; A. Charkhi y S. J. Ahmadi (2016). Nanocrystalline Zeolites in Superficial Water. Part A: Opportunities and Challenges for Synthesis Using Organic Templates. Journal for Supercritical Fluids, 102, 40-49.

Invernizzi, N.; G. Foladori; E. Robles-Belmont; E. Záyago; E. Arteaga; C. Bagattolli; T. Carroza; A. Chiancone y W. Urquijo (2015). Nanotecnologías dirigidas a necesidades sociales. Contribuciones de la investigación latinoamericana en medicina, energía y agua. Sociology and Technoscience, 5(2), 1-30.

Kharisov, B.I.; H.R. Diaz; O.V. Kharissova; V.M. Jiménez-Pérez; B.O. Pérez y B.M. Flores (2012). Iron-containing nanomaterials: synthesis, properties, and environmental applications. RSC Advances 2(25), 9325-9358.
Li, J.; J. Lin; X. Xu; X. Zhang; Y. Xue; J. Mi; Z. Mo; Y. Fan y X. Yang (2016). Porous Boron Nitride a High Surface Area: Hydrogen Storage and Water Treatment. Nanotechnology, 24(15).

Pendergast, M. y E. Hoek (2016). A Review of Water Treatment Membrane Nanotechnologies. Energy Environmental and Science, 4, 1946-1971.

Prachi, P.G.; D. Madathil y A.N.B. Nair (2016). Nanotechnology in Waste Water Treatment: A Review. International Journal of Chem Tech Research, 5(5), 2303-2308.

Pronovost, A. (2016). Nanotechnology Approach for Removing Hydrocarbons and Other Contaminants from Utility Vault and Substructure Wastewater. Remediation Journal, 26(3), 169-182.

Qu, X.; J. Brame; Q. Li y J.J. Álvarez (2013). Nanotechnology for a Safe and Sustainable Water Supply: Enabling Integrated Water Treatment and Reuse. Acc. Chem. Res., 46(3), 834-843.

Qu, X.; P. Álvarez y Q. Li (2016). Applications of Nanotechnology in Water and Wastewater Treatment. Water Research, 47(12), 3961-3946.

Rickerby, D.G. y M. Morrison (2016). Nanotechnology and the environment: A European perspective. Science and Technology of Advanced Materials, 8(1), 19-24.

Saldívar, L. y C. Walsh. (2015). Nanotecnología para el tratamiento de agua. Claves sobre la investigación en México. Mundo Nano, 8(14), 53-69.

Seul-Yi, L. y S. Park (2016). TiO2 Photo catalyst for Water Treatment Applications. Journal of Industrial and Engineering Chemistry, 19(6) (2013), 1761-1769.

Takeuchi, N. y M. Mora (2011). Divulgación y Formación en Nanotecnología en Méxi- 
co. Mundo Nano, 4(2). Recuperado de www. revistas.unam.mx/ index.php/nano/article/ view/44978 Consultado el 15 de agosto de 2016.

UNESCO (United Nations Educational, Scientific and Cultural Organization) (2015). Water for sustainable world. Recuperado de http://unesdoc.unesco.org/ images/0023/002318/231823E.pdf.

UN-Water (2016) Water and Jobs. The United Nations World Water Development Report 2016. Recuperado de http://unesdoc.unesco. org/images/ 0024/002439/243938e.pdf.
Vaseashta, A.; M. Vaclavikova; S. Vaseashta; G. Gallios; P. Roy y O. Pummakarnchana (2016). Nanostructures in environmental pollution detection, monitoring, and remediation. Science and Technology of Advanced Materials, 8, 47-59.

Záyago, E.; F. Stacey y G. Foladori (2014). Twelve years of nanoscience and nanotechnology publications in México. Journal of Nanoparticle Research, 16(2123), 1-10.

Záyago, E.; G. Foladori; S. Frederick y R. Arteaga (2015). ¿Se estudian los riesgos de los nanomateriales en México? Temas de Ciencia y Tecnología, 56(19), 17-27.

Recibido: 28 de febrero de 2018 Aceptado: 19 de abril de 2018 
Panorama de la investigación y desarrollo de las nanotecnologías para tratamiento de agua

\section{Anexos}

\section{Anexo 1}

Categorías y palabras clave utilizadas en la búsqueda de artículos sobre nanotecnología y agua

\begin{tabular}{|c|c|}
\hline Categorías & Palabras clave \\
\hline Remediación- Purificación & 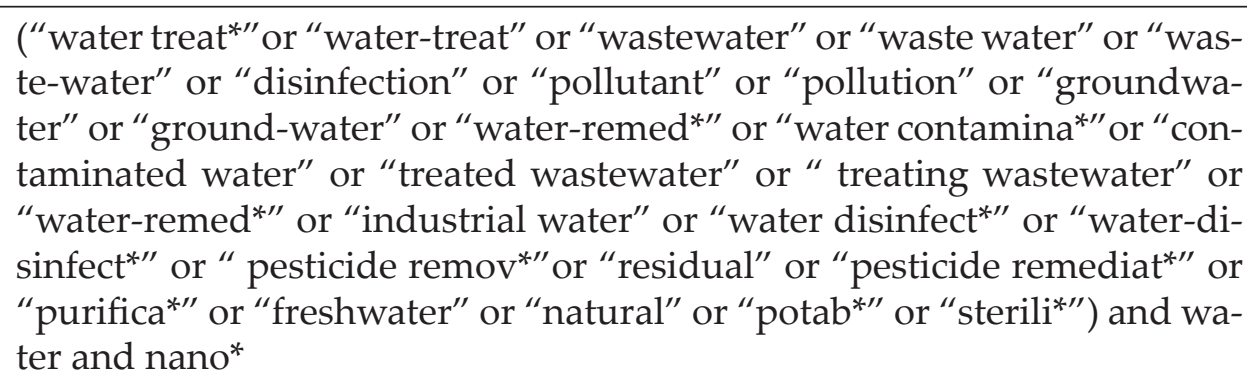 \\
\hline Detección-Filtración & $\begin{array}{l}\text { (("membrane filtration" or "dendrimer filtering" or "filtration" or "na- } \\
\text { nofiltration" or "nano-filtration" or "membrane system" or "reverse os- } \\
\text { mosis" or "electrodialysis" or "multiple stage flash distillation" or "acti- } \\
\text { vated carbon" or "drink") and water and nano*) }\end{array}$ \\
\hline Desalinización & $\begin{array}{l}\text { (("nanoporous polymeric materials") or ("brackish" or "desalination" or } \\
\text { "saltwater" or "salt water" or "salt-water" or "seawater" or "sea water" } \\
\text { or "sea-water") and nano*) }\end{array}$ \\
\hline
\end{tabular}

Anexo 2

Método de búsqueda para filtrar cuerpos académicos de PRODEP

\begin{tabular}{|c|c|c|c|}
\hline $\begin{array}{c}\text { 1.er filtro } \\
\text { Subsistema }\end{array}$ & $\begin{array}{c}\text { 2.do filtro } \\
\text { Área de conocimiento }\end{array}$ & $\begin{array}{l}\text { 3.er filtro } \\
\text { Grado de consolidación }\end{array}$ & $\begin{array}{l}\text { 4.to filtro } \\
\text { Palabras clave }\end{array}$ \\
\hline $\begin{array}{l}\text { a) Universidades públicas y } \\
\text { relacionadas } \\
\text { b) Universidades politécnicas } \\
\text { c) Universidades tecnológicas } \\
\text { d) Institutos tecnológicos } \\
\text { federales } \\
\text { e) Institutos tecnológicos } \\
\text { descentralizados } \\
\text { f) Escuelas normales }\end{array}$ & $\begin{array}{l}\text { a) Agricultura y pesca } \\
\text { b) Salud } \\
\text { c) Ciencias naturales e Ingeniería } \\
\text { d) Sociales y administrativas } \\
\text { e) Ingenierías y tecnologías } \\
\text { f) Educación, humanidades y artes }\end{array}$ & $\begin{array}{l}\text { Cuerpos Académicos } \\
\text { en Formación (CAEF) } \\
\text { Cuerpos Académi- } \\
\text { cos en Consolidación } \\
\text { (CAEC) } \\
\text { Cuerpos Académicos } \\
\text { Consolidados (CAC) }\end{array}$ & $\begin{array}{l}\text { Nanotecnología } \\
\text { y agua }\end{array}$ \\
\hline
\end{tabular}


J. Castañeda, G. Voladori, S. León, E. Robles-Belmont y E. Zágayo

Anexo 3

Palabras clave utilizadas en las búsquedas de patentes en el tratamiento de aguas con nanotecnologías en México

\begin{tabular}{lc}
\hline \multicolumn{1}{c}{ Categorías } & \multicolumn{1}{c}{ Palabras clave } \\
\hline Remediación- Purificación & $\begin{array}{l}\text { (nano* AND water AND (remediation) }) \\
(\text { nano* AND water AND (purification) })\end{array}$ \\
Detección-Filtración & $\begin{array}{l}(\text { nano* AND water AND (filtration) }) \\
(\text { nano* AND water AND(detection) })\end{array}$ \\
Desalinización & $($ nano* AND water AND (desalination) $)$ \\
\hline
\end{tabular}

